\title{
Editorial
}

\section{Espacios del género y poder}

\author{
Claudia Mora ${ }^{1}$ y Daniela Cáceres ${ }^{2}$ \\ Coordinadoras Dossier: Género, poder y desigualdades
}

Revista Temas Sociológicos, N²2, 2018.

Este número especial de la Revista Temas Sociológicos aborda las relaciones de poder constitutivas del género en los procesos de organización y distribución de recursos sociales en el mercado del trabajo; en las significaciones que condicionan la incorporación de sus asuntos y demandas en la agenda pública, y en regímenes sociopolíticos que contribuyen a la violencia de género, una de las manifestaciones más crudas de la jerarquía y desigualdad en este ámbito. En este dossier, titulado "Género, Poder y Desigualdades", el conjunto de trabajos seleccionados forma parte del núcleo reflexivo de las ciencias sociales acerca del género y feminismos, y plantea la discusión acerca de los efectos subjetivos y sociales de la diferenciación genérica; los contextos sociopolíticos en que se desarrollan relaciones de género/poder, así como sus múltiples manifestaciones cotidianas.

Este número, convocado meses antes del llamado "mayo feminista" chileno, viene a iluminar la prolífica reflexión acerca

Doctora en Sociología por la Northeastern University, Estados Unidos. Investigadora y profesora de la Universidad Academia de Humanismo Cristiano, Chile. Contacto: claudiamoradelvalle@gmail.com

2 Magíster en Análisis Sistémico Aplicado en la Sociedad, por la Universidad de Chile, Chile. Doctorante en Sociología por la Universidad Alberto Hurtado, Chile. Contacto: dcaceres@uahurtado.cl 
de los procesos de producción de la diferencia de género que, invisibles por su cotidianeidad, socavan los esfuerzos democratizadores que los movimientos sociales han impulsado en Chile -y en la región- en las últimas décadas (Mora, 2013). La presión de los movimientos recientes en el país reveló la arbitrariedad y violencia del género como un aparato de producción y normalización de la feminidad masculina (Butler, 2004), generando prometedores compromisos institucionales. No obstante, el impulso reaccionario (backlash) que usualmente acompaña los desafíos a grupos que monopolizan recursos y las estructuras de poder (Mansbridge y Shames, 2008), se fundamenta, a menudo, en la legitimidad de un orden natural de género, que vive en las prácticas cotidianas de los sujetos.

Los sistemas social, económico y político desarrollados en relación con los intereses de grupos con poderosos no requieren de la anuencia o, siquiera, de la conciencia del privilegio de algunos; ni de la fuerza sobre otros para lograr que actúen en contra de sus propios intereses. Sin embargo, la pérdida de poder activa resistencias, expresadas en violencia y descrédito, y, más omnipresente, en guerra cultural: reclamaciones acerca de lo excesivo de los cambios, a su desmedida rapidez y, fundamentalmente, en relación con la necesidad de preservar valores sociales que definen e identifican la nación (ibíd). Esta batalla se fragua en las prácticas cotidianas de diferenciación de género, en las que las pugnas por justicia social se enmarcan, a menudo, en desafíos netamente valóricos.

Los espacios de reproducción de la diferencia, así como los procesos que reiteran las desventajas grupales de las mujeres, son abordados en este número especial de Revista Temas Sociológicos enfocándose en tres dimensiones centrales: mercado laboral, violencia de género y transgresión normativa al significante mujer. Los primeros tres artículos proporcionan un análisis acerca de los regímenes de género que regulan la incorporación y movilidad laboral de las mujeres en Chile. El mercado del trabajo chileno es una de las instituciones que refleja más claramente las desigual- 
dades sociales, en tanto ubica recursos -ingreso, autoridad, prestigio, por ejemplo- en virtud de criterios formales, como el capital cultural acumulado, pero, principalmente, en virtud de criterios informales, como la membresía en grupos sociales específicos. El género, la clase social y el origen nacional, entre otros, condicionan la posición de los trabajadores en la estructura ocupacional, y, en el caso de las mujeres, su posición en estas estructuras refleja patrones de exclusión, en tanto las concentra en ciertas actividades y jerarquías ocupacionales (Valenzuela y Mora, 2009).

Las contribuciones a este número dan cuenta de casos de estudio específicos en dos ramas de actividad -minería y servicios-, abordando en primer lugar las limitaciones de las políticas públicas e institucionales para la incorporación de las mujeres en el trabajo; luego, las implicancias que la igualdad de género tiene en las trayectorias laborales de mujeres profesionales y universitarias y, tercero, la significación de los liderazgos femeninos en la visión de las propias mujeres. De este modo, esta sección del dossier aporta a la discusión de algunos aspectos fundamentales para la compresión de los mecanismos de exclusión del trabajo: las limitaciones de las políticas de inclusión frente a la significación del género de trabajadoras/es y empleadores/as que las desafían; la reiteración de la vinculación entre lo reproductivo con las expectativas y evaluación del liderazgo femenino, y la injerencia de las conceptualizaciones subjetivas en la identidad de "trabajadora" de las mujeres chilenas.

El primer artículo, titulado "Equidad de género en la gran minería del cobre de Chile. Experiencias de inserción laboral femenina en espacios masculinizados", de Gómez y Angelcos, plantea que, a pesar de la implementación de políticas públicas e institucionales de fomento a la integración de mujeres en el rubro, persisten, como barreras fundamentales a la incorporación de las mujeres en la minería, fronteras sociales que delimitan la segregación de las mujeres a trabajos estereotipados por género. Los autores analizan cómo los estereotipos laborales asociados a lo masculino y femenino, y la vinculación entre mujer y madre, 
definen las actividades y labores de hombres y mujeres al interior de la actividad minera, privilegiando distinciones referidas a los cuerpos y a capacidades intelectuales "innatas".

A partir de veinte entrevistas en profundidad a trabajadoras en las divisiones El Teniente y Chuquicamata, Gómez y Angelcos muestran que estereotipos asociados al trabajo femenino y masculino constituyen fronteras simbólicas que permiten la integración y legitimación de la mujer en la actividad minera en espacios acordes con la definición simbólica de "lo femenino", por ejemplo, en las labores de gestión y administración en la empresa minera. Pero que los mismos estereotipos refuerzan la segregación de las mujeres en el caso del trabajo en terreno. Plantean los autores que "el traslado de lógicas familiares al interior del trabajo -basadas en una relación paternalista entre trabajadores varones con trayectoria y mujeres jóvenes recién ingresadas-facilita la relación entre distintos géneros, al mismo tiempo que reproduce la posición de subordinación que tienen las mujeres". Esta relación de subordinación es reiterada por hombres y mujeres en la insistencia del rol maternal primario de las mujeres. La atribución exclusiva del cuidado y labores reproductivas a las mujeres trabajadoras contribuye a "una pretensión de inmutabilidad de los roles dispuestos en la familia, por lo que la maternidad constituye una frontera simbólica que limita las trayectorias de inserción femenina y desarrollo en el rubro minero". Concluyen los autores que, "a pesar de los avances declarados por las trabajadoras de la División Chuquicamata y de los retrasos expresados en la División El Teniente, las políticas institucionales de las empresas mineras aún se presentan como formas de solucionar problemáticas de "las mujeres" o de "las madres", excluyendo a la población masculina en la responsabilidad de la crianza y la familia, reforzando con ello el imaginario de género".

Un aporte importante a la discusión relativa a la incorporación de las mujeres al mercado laboral chileno es desarrollado por la antropóloga Carmen Gloria Godoy, quien plantea la relevancia de los significados atribuidos a la igualdad de género en la 
configuración genérica de mujeres profesionales y universitarias jóvenes. A partir de 25 entrevistas a mujeres de capas medias y altas, Godoy analiza la centralidad de una noción de igualdad de género en la construcción de sus proyectos de vida y en sus prácticas cotidianas, en el ámbito familiar, educativo y laboral.

El horizonte normativo que inspira las prácticas cotidianas de las mujeres en el mercado del trabajo demuestra ser un factor determinante en las trayectorias laborales de las mujeres, en la medida en que configura un "tipo ideal" en relación al cual las performances individuales son evaluadas. Plantea la autora que, aun cuando "la situación de las mujeres, en general, había mejorado en relación con la experiencia de sus madres y abuelas. No obstante, esa igualdad se desplegaba de distinto modo si se trataba de la familia, el trabajo o los estudios, así también de acuerdo con sus propias historias familiares". La "herencia familiar" en relación a la importancia de la igualdad de género incide de manera determinante en "la posibilidad de generar un proyecto de vida propio (ya sea orientado al desarrollo profesional, la maternidad $\mathrm{u}$ otros proyectos personales), en el que la independencia económica adquiere centralidad, aparece como una de las principales transformaciones relativas a la concreción de la igualdad de género, así como la posibilidad y el deseo de ser exitosas profesionalmente".

La autora destaca la intersección de la clase social en la percepción del lugar ocupado en las esferas familiares, educacionales y laborales de las mujeres en Chile, haciendo ver que la sensación de inadecuación que acompaña a las mujeres puede no ser experimentada por quienes cuentan con mayores recursos económicos. Plantea Godoy que "la posibilidad de acceder a estudios superiores, impulsadas por sus propios padres y teniendo como modelo a una madre trabajadora, para desarrollar una carrera profesional, configura un horizonte en el cual el trabajo se vuelve constitutivo de la subjetividad -sin desplazar del todo a la maternidad-resistiéndose así a las definiciones externas respecto de lo que significa ser una mujer y sus límites de actuación". 
Finalmente, el trabajo de Sánchez y Villarroel profundiza en la conceptualización del liderazgo femenino ejercido en el trabajo. Destacando la baja participación laboral femenina en el concierto global, los autoras dan luces sobre las presunciones que acompañan a las mujeres que sí participan del mercado laboral, ejerciendo roles de autoridad. En concordancia con la literatura, Sánchez y Villarroel muestran que el valor atribuido al liderazgo de las mujeres continúa basándose en capacidades estereotípicamente femeninas. Plantean los autoras que las entrevistas permiten "observar un reconocimiento y valoración positiva de lo que socialmente sería eminentemente femenino, atribuyendo al liderazgo características propias del "ser mujer". Esto aportaría una visión distinta de la masculina, señalando que su presencia permitiría transformar la forma de direccionar personas a su cargo y el desarrollo de las organizaciones en las que participan, mirada que principalmente emerge desde quienes ejercen las jefaturas femeninas".

Los autores destacan que, en el ejercicio de la autoridad, el rango etario de las mujeres determina un estilo de liderazgo más autoritario entre aquellas de mayor edad, versus un estilo más ideológico entre las más jóvenes. No obstante, la participación de las mujeres en el trabajo y en roles de liderazgo sigue estando condicionada por presunciones acerca de sus habilidades "naturales" por la mediación, el diálogo, y trabajo en equipo, en lo que la literatura ha denominado "formas maternalistas de liderazgo".

Los aportes de este dossier al conocimiento acerca de las dinámicas del mercado laboral que permean -y limitan- la participación de las mujeres en el trabajo productivo, profundizan un análisis que da cuenta de la persistencia de la definición de lo femenino como ligado a lo reproductivo y como inherentemente distinto del aporte masculino: las "cualidades blandas" de las mujeres equilibrarían la competitividad y autoritarismo propio de los hombres. La insistencia en la definición genérica de los trabajadores es uno de los factores clave que previene la configuración del mercado laboral chileno como una vía concreta e ínte- 
gra de movilidad social, y como un mecanismo de distribución equitativo de recursos sociales.

Los efectos materiales y simbólicos del cruce de múltiples desigualdades sociales son analizados transversalmente desde la aproximación interseccional (Crenshaw, 1989, 1991) en los espacios creados por el género, territorio y clase social. En efecto, el género y la construcción de un sistema social sexo/género (Rubin, 1996), binario y heteronormado, condiciona las experiencias de vida así como los imaginarios colectivos de hombres y mujeres (Nash, 2014). Cuando el género se conjuga con otras variables, tales como la clase social o la identidad sexual -que subvierte la binariedad antes mencionada- funda nuevas condiciones de vulnerabilidad que pueden implicar segregación, exclusión social y violencias múltiples.

Respecto de la(s) violencia(s) -uno de los ejes que articulan este número-, si bien la institucionalidad ha ofrecido definiciones sobre violencia de género, y contra la mujer en particular, como un fenómeno transversal a mujeres y niñas, es necesario distinguir distintas manifestaciones y gradientes de exposición a la violencia en virtud de la intersección de desigualdades sociales, por ejemplo, mujeres, pobres, inmigrantes, de color, lesbianas, minusválidas u otros. Dicho de otro modo, la violencia contra las mujeres se relaciona con otras formas de violencia relativas a condiciones sociales, como la pobreza, la marginación, la explotación económica, u otras (Castañeda, Ravelo y Pérez, 2013, p. 13).

Las particularidades geográficas en el marco de las relaciones de género ofrecen una lectura respecto de las condiciones de existencia de las mujeres en distintos lugares del globo. Por ello, es de especial significancia la inclusión, en el eje de violencia de género, de dos investigaciones realizadas en México, uno de los países con las tasas más altas de violencia contra la mujer y feminicidio/femicidio de América Latina. Ambos artículos tienen en común una postura crítica frente a la Ley General de Acceso de las Mujeres a una Vida Libre de Violencia (LGAMVLV), promulgada en México en 2007, presentando cifras de mujeres víctimas 
de violencias múltiples -pese a los vacíos estadísticos al respecto- $y$ graficando las resistencias institucionales para ejecutar mecanismos de prevención. Ambas autoras plantean que el machismo, como dimensión estructural, dificulta la eficacia de dicha ley.

En consideración a la complejidad de la violencia de género, el trabajo de Graciela Vélez y Héctor Serrano contribuye a la reflexión sobre el fenómeno desde la multiplicidad de factores que inciden en la violencia, además de analizar el trabajo de prevención y erradicación por parte del Estado y la política pública. El artículo, a partir de un diagnóstico contextual y de entrevistas a hombres procesados judicialmente por maltrato y/o violencia de género, distingue elementos que permiten visualizar nodos críticos que impiden avanzar en términos de prevención, tales como la falta de enfoque de género institucional y la resistencia a incorporarlo. De esta manera, sobre la base del "modelo ecológico" desarrollado por Bronfenbrenneren, los autores proponen un análisis integral de la violencia de género y, en consecuencia, un diseño de políticas públicas que aborde la prevención pensando en instrumentos con enfoque de género que también estén orientados a generar condiciones de igualdad entre hombres y mujeres.

Por otra parte, el artículo presentado por Rocío Rosas propone un análisis sobre la violencia feminicida en el Estado de Guanajuato y sus implicancias en la vida de las mujeres. Para ello, la metodología utilizada es el análisis de fuentes documentales, a través de la sistematización de feminicidios con aparición en periódicos locales y el análisis de datos secundarios relativos a violencia de género. En virtud de la convergencia del análisis presentado, el trabajo propone diferentes argumentos para explicar el feminicidio, realizando una revisión conceptual y taxonómica sobre tipos de violencia hacia la mujer, complementado con definiciones de feminicidio a nivel institucional/legal y académico. Identifica, además, las características socioculturales de México, país cuya estructura patriarcal perpetúa los roles tradicionales de género, la subordinación de las mujeres y, con ello, la violencia 
hacia la mujer. Este trabajo es de especial relevancia práctica por la falta de datos sistematizados en el lugar, pese al incremento de delitos contra mujeres en el sector del "corredor industrial", emplazado en el Estado de Guanajuato.

La discusión sobre la violencia, en tanto problema macro y micro que se visualiza en el plano estructural y en el de la interacción social, tiene su génesis en las relaciones asimétricas de poder entre hombres y mujeres, y en el lugar de postergación y subordinación al género femenino. Existe una vasta literatura sobre la distinción público/privado en tanto espacios generizados y de distinto valor: hombres/público y mujeres/privado, reflejado en dicotomías como la política y las labores de reproducción, o el trabajo remunerado y el trabajo doméstico (no remunerado). En este contexto, la violencia viene a subrayar su trasfondo relacional -en tanto relación de poder-. En el orden patriarcal, la violencia contra la mujer evidencia el intento por la restauración del orden que se ve amenazado (Cáceres, 2016) y sus expresiones simbólica, económica y física, así como la más extrema, el femicidio, proyectan señales de alerta que las ciencias sociales no pueden ignorar.

En otra dimensión de análisis, el soporte heteronormativo en la diferenciación del espacio social público/privado configura un régimen institucional construido en torno a la diferencia, que condiciona las posibilidades de existencia de "subjetividades transgresoras". Un tercer eje de este número aborda los desafíos implicados en la subversión a la norma y cómo dicha transgresión tiene efectos en los ámbitos de la experiencia, las motivaciones y las representaciones.

El artículo presentado por Paola Contreras aborda las consecuencias personales, familiares y sociales que experimentan las mujeres al vivir parte de sus maternidades encarceladas. La autora enfatiza la vivencia de estas mujeres como una doble condena: social y penal. Fundamentalmente, porque son mujeres cuya infracción sobrepasa el ámbito penal al contravenir el binomio ontologizado de mujer-madre y, en consecuencia, al constituirse 
como "malas madres", juzgadas y sancionadas socialmente. El artículo hace hincapié en las lógicas del sistema penitenciario que reproducen los roles de género y complejizan la experiencia de la maternidad. De esta manera, la historiadora propone, desde el análisis del discurso crítico feminista, una reflexión sobre una serie de entrevistas realizadas a mujeres que estuvieron privadas de libertad en Barcelona, España, mientras vivían la maternidad, para luego, desde los hallazgos, abrir un debate que "desmantele" los prejuicios y estereotipos que estas mujeres cargan.

En el ámbito de la participación política, en el marco de las luchas por el reconocimiento y los derechos sexuales y reproductivos de las disidencias sexuales, las sociólogas Catalina Otárola y Catalina Tapia nos presentan una investigación basada en el análisis de las motivaciones y experiencias, en tanto dimensiones mutuamente implicadas, que impulsan a mujeres lesbianas a participar en diferentes organizaciones del movimiento LGBTI+ en Chile. El artículo parte del supuesto que, desde el género, se despliegan relaciones de poder asimétricas que influyen en las experiencias de los sujetos/as y, en este sentido, el objeto de estudio se implica en un doble registro: el de la experiencia vital y el de la participación política de mujeres lesbianas, ambos en un mundo liderado históricamente por hombres. De esta manera, mediante un enfoque cualitativo, el artículo nos brinda la posibilidad de conocer las motivaciones desde una lógica que desborda lo racional para enfatizar en la importancia de las emociones y los valores como vectores de participación política.

El análisis realizado por las autoras es un aporte a las investigaciones existentes sobre movimientos sociales subalternos que han despertado un especial interés en el seno de las epistemologías feministas, fundamentalmente por los procesos de subalternización desarrollados al alero de agendas políticas más amplias, en las cuales el género y las diversas dimensiones implicadas en esta categoría analítica terminan en un espacio de opacidad en el marco de la interseccionalidad política (Walby, Armstrong y Strid, 2012; Crenshaw, 1991). 
Los tres ejes temáticos del número especial que aquí presentamos -mercado laboral, violencia de género y transgresión normativa al significante mujer- contribuyen a evidenciar, a partir de casos de estudio empíricos, que la construcción genérica es siempre relacional, sociohistórica y atravesada por el poder. Como planteara Joan Scott hace más de tres décadas, el género es uno de los principales campos de articulación de poder, y un facilitador de la significación del poder a través de su construcción mutua (1986).

Finalmente, este dossier se cierra con dos trabajos relativos a las dos secciones complementarias para conformar el corpus completo de la Revista Temas Sociológicos. El artículo de Juan Pablo Venables, "Sociología y Opinión Pública: Desmontando un dispositivo", reflexiona acerca de formas de poder que construyen realidades y agendas públicas. El artículo constituye un aporte significativo a la comprensión de la opinión pública en tanto artefacto construido por las encuestas de opinión, las cuales legitiman una cierta política y consolidan las relaciones de fuerza que la hacen posible. Venable releva la necesidad de considerar en las encuestas no solo las creencias y opiniones de los individuos, sino que, además, los "constreñimientos, sanciones o restricciones sobre ellos, así como el aspecto material expresado en acciones o prácticas que dan su especificidad a la realidad social". Así, el autor da luces acerca de la necesidad de explorar no solo estadísticas y opiniones de los sujetos, sino que de dilucidar los procesos sociopolíticos, culturales y económicos que condicionan sus percepciones, prácticas y posiciones sociales.

Para concluir, la reseña escrita por las sociólogas Javiera Osses y Daniela Valencia, en referencia al libro "Ser Política en Chile", de la socióloga y cientista política Julieta Kirkwood, sitúa en este número especial una de las obras más influyentes para el feminismo en Chile. A poco más de treinta años de su publicación, el análisis de la relación entre mujeres y política, así como la invisibilidad y subalternidad de las mujeres en una serie de espacios sociales, parece una lectura aún vigente $\mathrm{y}$, en este sen- 
tido, el aporte de esta reseña al dossier, recordando a una de las mujeres más significativas en el campo de los estudios de género y la mujer, es un homenaje a su contribución.

En consideración a que el género es una dimensión de desigualdad social que se materializa en condiciones de existencia, la apuesta de este dossier es relevar los estudios sociales de género que, sustentados desde un paradigma crítico, alcancen en Chile, en el debate académico y político, el lugar que tanto la multiplicidad de centros de estudio e investigación, así como el sistema internacional de derechos humanos les han otorgado. Cada vez más los movimientos de mujeres y LGBT+ colorean las calles del mundo, poniendo de relieve demandas históricas habitualmente opacadas. Los movimientos feministas de Chile 2018 advierten sobre las experiencias de desigualdad de mujeres y minorías sexuales, desafiando las resistencias institucionales a la incorporación de la perspectiva de género, los discretos protocolos contra el acoso sexual en las instituciones universitarias, y las menguadas leyes en materia de derechos humanos de las mujeres y las minorías sexuales, dando cuenta, con ello, de la necesidad de poner el foco en las relaciones entre el género, el poder y las desigualdades sociales.

\section{Referencias}

Butler, J. (2004). Undoing gender. Nueva York: Routledge.

Cáceres, D. (2016). Sobre la semántica del femicidio en Chile. Sociedad y economía, 239-262.

Castañeda, M., Ravelo, P. y Pérez, T. (2013). Feminicidio y violencia de género en México: omisiones del Estado y exigencia civil de justicia. Iztapalapa Revista de Ciencias Sociales y Humanidades, 74, 11-39.

Crenshaw, K. (1989). Demarginalizing the intersection of Race and Sex: A black Feminist Critique of Antidiscrimination Doctrinem Feminist Theory and Antiracist Politics. University of Chicago Legal Forum, 138-167. 
Crenshaw, K. (1991). Mapping the Margins: intersectionality, identity Politics, and Violence against Women of Color. Stanford Law Review, 1241-1299.

Mansbridge, J. \& Shames, S. L. (2008). Toward a theory of backlash: Dynamic resistance and the central role of power. Politics $\mathcal{E}$ Gender, 4 (4), 623-634.

Mora, C. (2013). Desigualdad en Chile: la continua relevancia del género. Santiago de Chile: Ediciones Universidad Alberto Hurtado.

Nash, M. (Ed). (2014). Feminidades y masculinidades. Madrid: Alianza Editorial.

Rubin, G. (1996). El tráfico de mujeres: notas sobre la "economía política" del sexo, en M. Lamas, El género: la construcción cultural de la diferencia sexual (pp. 35-96). México D.F.: PUEG, Miguel Ángel Porrúa.

Scott, J. (1996). El género como categoría de análisis histórico, en M. Lamas, El género: la construcción cultural de la diferencia sexual (pp. 265-302). México D.F.: PUEG, Miguel Ángel Porrúa.

Valenzuela, M. E. \& Mora, C. (2009). Trabajo doméstico: un largo camino hacia el trabajo decente. Santiago de Chile: Organización Internacional del Trabajo.

Walby, S., Armstrong, J. \& Strid, S. (2012). Instersectionality: Multiple inequalities in Social Theory. Sociology, 224-240. 